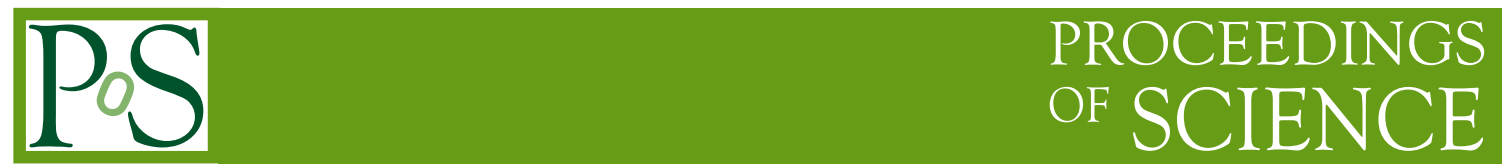

\title{
Bottom and strange Yukawa couplings at the NNLO in the MSSM
}

\section{Margherita Ghezzi*}

Institut für Theoretische Physik, Eberhard Karls Universität Tübingen, Germany

E-mail: margherita.ghezzi@itp.uni-tuebingen.de

The bottom and strange Yukawa couplings can play an important role in Higgs phenomenology in MSSM scenarios characterized by large $\tan \beta$. In these Proceedings we will review the current knowledge of the radiative corrections to these Yukawa couplings the main features of the NNLO calculation.

ALPS 2019 An Alpine LHC Physics Summit

April 22 - 27, 2019

Obergurgl, Austria

* Speaker. 


\begin{tabular}{|c|c|c|c|c|}
\hline \multicolumn{2}{|l|}{$\Phi$} & $g_{u}^{\Phi}$ & $g_{d}^{\Phi}$ & $g_{V}^{\Phi}$ \\
\hline SM & $H$ & 1 & 1 & 1 \\
\hline MSSM & $h$ & $\cos \alpha / \sin \beta$ & $-\sin \alpha / \cos \beta$ & $\sin (\beta-\alpha)$ \\
\hline & $H$ & $\sin \alpha / \sin \beta$ & $\cos \alpha / \cos \beta$ & $\cos (\beta-\alpha)$ \\
\hline & $A$ & $1 / \tan \beta$ & $\tan \beta$ & 0 \\
\hline
\end{tabular}

Table 1: Couplings of the Higgs bosons to SM particles in the MSSM.

\section{Introduction}

In the Minimal Supersymmetric extension of the Standard Model (MSSM) the Higgs sector consists in five elementary Higgs bosons, contained in two complex scalar doublets: two neutral CP-even bosons $h$ and $H$, one neutral CP-odd boson $A$ and two charged bosons $H^{ \pm}$. Their couplings to fermions and vector bosons, which are reported in Table 1, depend on the mixing angles $\alpha$ and $\beta$, which are defined by the diagonalization of the neutral and charged Higgs mass matrices. In scenarios characterized by large $\tan \beta \equiv v_{2} / v_{1}$ (with $v_{1}$ and $v_{2}$ tbeing the vacuum expectation values (VEVs) of the two Higgs doublets), the down-type Yukawa couplings are generally strongly enhanced, while the up-type ones are strongly suppressed. As a consequence, processes induced by the bottom Yukawa couplings become dominant in this kind of scenarios. This is the case, in particular, of Higgs decays into bottom quarks and Higgs bremsstrahlung off bottom quarks. The bottom loop contribution to the Higgs production via gluon fusion acquires relevance as well.

For this reason, a precise evaluation of the down-type quark Yukawa couplings is particularly important. In these Proceedings we will review the state-of-the-art of the calculation of the SUSY-QCD corrections, that are currently known up to the NNLO [1-3], and resummed via an effective-field-theory approach $[4,5]$. The supersymmetric electroweak (EW) corrections are currently known at the 1-loop level [6-8], while the calculation of their SUSY-QCD corrections, of $\mathscr{O}\left(\alpha \alpha_{s}\right)$, is currently work-in-progress [9]. In analogy with the bottom quark case, also the strange effective Yukawa couplings have been calculated up to the NNLO in SUSY-QCD [3]. This calculation plays a role in particular in the subleading decay mode of the charged Higgs $H^{+} \rightarrow c \bar{s}$.

\section{Effective bottom and strange Yukawa couplings}

Following an effective approach, the radiative corrections to the Yukawa couplings $\Delta_{q, 1}$ and $\Delta_{q, 2}$ of the two Higgs doublets $\phi_{1}^{0}$ and $\phi_{2}^{0 *}$ can be encoded into a single factor $\Delta_{q}$, as shown in the effective Lagrangian

$$
\begin{aligned}
\mathscr{L}_{e f f} & =-\sum_{q=b, s} \lambda_{q} \bar{q}_{R}\left[\left(1+\Delta_{q, 1}\right) \phi_{1}^{0}+\Delta_{q, 2} \phi_{2}^{0 *}\right] q_{L}+\text { h.c. } \\
& =-\sum_{q=b, s}\left[\frac{m_{q}}{1+\Delta_{q}} \bar{q}_{R} q_{L}+\frac{m_{q}}{v_{1}\left(1+\Delta_{q}\right)} \bar{q}_{R}\left(H_{1}+\frac{\Delta_{q}}{\tan \beta} H_{2}\right) q_{L}\right]
\end{aligned}
$$

with $\Delta_{q} \equiv \Delta_{q, 2} \tan \beta /\left(1+\Delta_{q, 1}\right)$ and the neutral components $\phi_{1}^{0}$ and $\phi_{2}^{0 *}$ of the two Higgs doublets expanded around the VEVs, $\phi_{1}^{0}=H_{1}+v_{1}, \phi_{2}^{0 *}=H_{2}+v_{2}$. 


\section{Details of the Calculation}

At a practical level, the calculation [1-4] was carried out with the help of the Low Energy Theorems $[10,11]$ : any matrix element with an external Higgs field can be related to the corresponding matrix element without the external Higgs in the limit of vanishing Higgs momentum, through the replacement

$$
\begin{aligned}
& v_{1} \rightarrow \sqrt{2} \phi_{1}^{0}, \\
& v_{2} \rightarrow \sqrt{2} \phi_{2}^{0 *} .
\end{aligned}
$$

Therefore, the calculation of the three-point functions giving $\Delta_{b, s}$ is reduced to the calculation of the bottom and strange self energies. In particular, it turns out that the leading contributions to $\Delta_{b, s}$ come from the scalar part $\Sigma_{S}\left(m_{q}^{2}\right)$ of the self energy

$$
\Sigma_{S}\left(m_{q}^{2}\right)=\frac{\lambda_{b}}{\sqrt{2}} v_{1}\left[\Delta_{q, 1}+\Delta_{q, 2} \tan \beta\right],
$$

that also modifies the relation between the pole mass and the Yukawa coupling beyond the tree level:

$$
m_{q}=\frac{\lambda_{q}}{\sqrt{2}} v_{1}+\Sigma_{S}\left(m_{q}^{2}\right)
$$

At the one-loop level, the contributions to $\Delta_{q, 2}$ can be split into QCD and EW corrections:

$$
\Delta_{q, 2}=\Delta_{q, 2}^{\mathrm{QCD}}+\Delta_{q, 2}^{\mathrm{eW}}
$$

where $\Delta_{q, 2}^{\mathrm{QCD}}$ and $\Delta_{q, 2}^{\mathrm{ew}}$ can be derived from the bottom self energies containing an off-diagonal insertion in the sbottom and stop propagators, as shown in Figure 1. The off-diagonal contributions to the squark propagators can be read off the expression of the sfermion mass matrix in the current eigenstate basis:

$$
\mathscr{M}_{\tilde{f}}^{2}=\left(\begin{array}{c}
M_{L L}^{2} M_{L R}^{2} \\
M_{R L}^{2} M_{R R}^{2}
\end{array}\right)=\left(\begin{array}{cc}
M_{\tilde{f}_{L}}^{2}+m_{f}^{2} & m_{f}\left(A_{f}-\mu r_{f}\right) \\
m_{f}\left(A_{f}-\mu r_{f}\right) & M_{\tilde{f}_{R}}^{2}+m_{f}^{2}
\end{array}\right)
$$

where $r_{d}=1 / r_{u}=\tan \beta$ for down- and up-type sfermions and $A_{f}$ are the trilinear scalar couplings of the soft SUSY breaking terms.

The result of these 1-loop diagrams is finite and no renormalization procedure is in need. The expressions for $\Delta_{q, 2}^{\mathrm{QCD}}$ and $\Delta_{q, 2}^{\mathrm{ew}}$ are given in Eq. 4.4 for the bottom quark. They resum all the leading terms of order $\mathscr{O}\left[\left(\alpha_{s} \mu \tan \beta\right)^{n}\right]$ and $\mathscr{O}\left[\left(\lambda_{t}^{2} A_{t} \tan \beta\right)^{n}\right]$. Moreover, one can see from the off-diagonal terms of Eq. 3.5 that also the non-tan $\beta$-enhanced terms of order $\mathscr{O}\left[\left(\alpha_{s} A_{b}\right)^{n}\right]$ can be resummed at the same time. Similarly, the resummed strange Yukawa coupling contains all the terms of $\mathscr{O}\left[\left(\alpha_{s} \mu \tan \beta\right)^{n}\right]$ and $\mathscr{O}\left[\left(\alpha_{s} A_{s}\right)^{n}\right]$ at the one-loop level, while $\Delta_{s}^{\text {ew }}=0$.

\section{Calculation at the NNLO}

The NNLO SUSY-QCD corrections to $\Delta_{b, s}$ can be determined through the calculation of the 2-loop contributions to the bottom and strange self energies. More in detail, the relevant contributions 

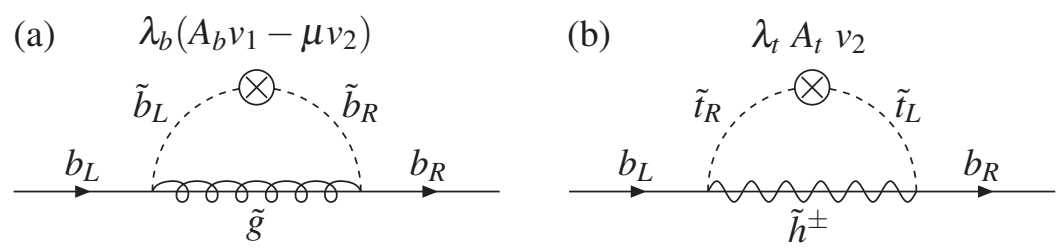

Figure 1: One-loop diagrams of (a) the SUSY-QCD and (b) the top-induced SUSY-EW contributions to the bottom self energy with the $M_{L R}^{2}$ mass insertions, giving the $\Delta_{b}$ at NLO [2].
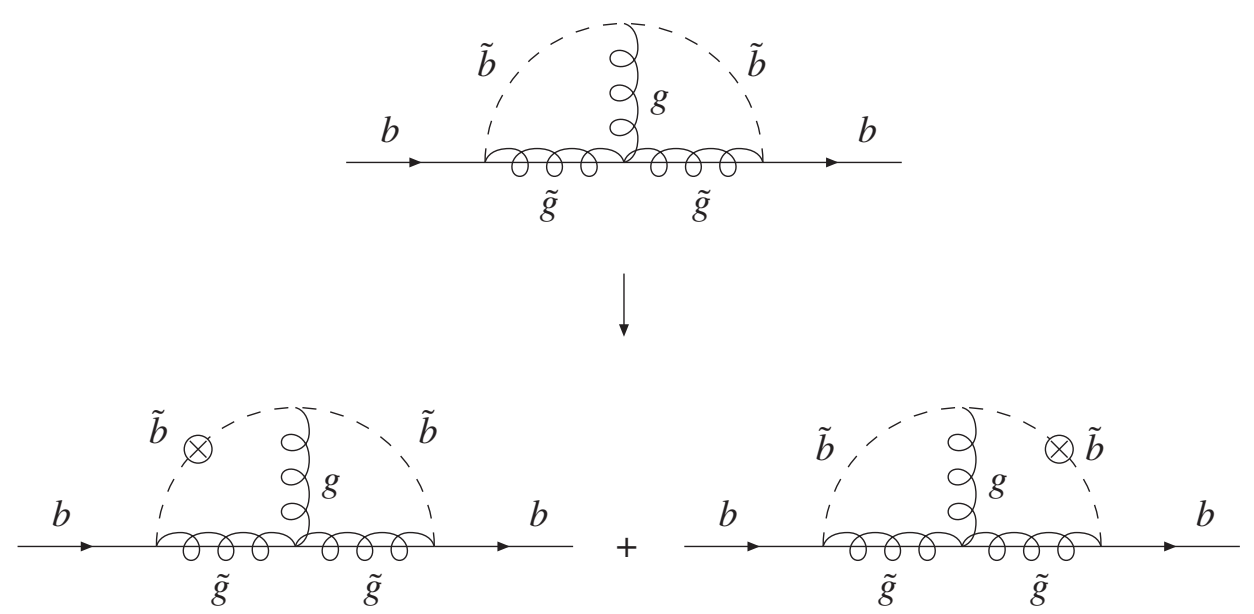

Figure 2: All possible mass insertions into one of the two-loop diagrams contributing to $\Delta_{b}$ at NNLO [2].

come from the two-loop diagrams containing one $M_{L R}^{2}$ insertion, as shown in Figure 2. Differently from the 1-loop calculation, the 2-loop Feynman diagrams give a divergent result, that needs to be renormalized. The result can be written in terms of the two following master integrals:

$$
A_{0}(m)=\bar{\mu}^{4-n} \int \frac{d^{n} k}{(2 \pi)^{n}} \frac{1}{k^{2}-m^{2}}
$$

and

$$
T_{134}\left(m_{1}, m_{3}, m_{4}\right)=\bar{\mu}^{2(4-n)} \int \frac{d^{n} k}{(2 \pi)^{n}} \frac{d^{n} q}{(2 \pi)^{n}} \frac{1}{\left(k^{2}-m_{1}^{2}\right)\left[(k-q)^{2}-m_{3}^{2}\right]\left(q^{2}-m_{4}^{2}\right)}
$$

where $n=4-2 \varepsilon$ and $\bar{\mu}$ denotes the 't Hooft mass. The renormalization procedure for $\Delta_{b, 2}$ goes along the lines of Ref [2]. The extension to the non- $\tan \beta$-enhanced terms proportional to $A_{b}$ (i. $e$. the calculation of $\left.\Delta_{q, 1}\right)$ does not require any further action on the renormalization side, because the parameter $A_{b}$ is not renormalized at $\mathscr{O}\left(\alpha_{s} A_{b}\right)$ (i.e. $\delta A_{b} \neq \mathscr{O}\left(A_{b}\right)$ ) [3].

The NNLO expression for $\Delta_{b}$ is then given by

$$
\Delta_{b}=\frac{\left(\Delta_{b, 2}^{Q C D}\left[1+\delta_{1}\right]+\Delta_{b, 2}^{e l w}\left[1+\delta_{2}\right]\right) \tan \beta}{1+\Delta_{b, 1}\left[1+\delta_{1}\right]}
$$

with the 1-loop contributions

$$
\Delta_{b, 1}=-\frac{C_{F}}{2} \frac{\alpha_{s}\left(\mu_{R}\right)}{\pi} m_{\tilde{g}} A_{b} I\left(m_{\tilde{b}_{1}}^{2}, m_{\tilde{b}_{2}}^{2}, m_{\tilde{g}}^{2}\right)
$$




$$
\begin{aligned}
\Delta_{b, 2} & =\Delta_{b, 2}^{Q C D}+\Delta_{b, 2}^{e l w} \\
\Delta_{b, 2}^{Q C D} & =\frac{C_{F}}{2} \frac{\alpha_{s}\left(\mu_{R}\right)}{\pi} m_{\tilde{g}} \mu I\left(m_{\tilde{b}_{1}}^{2}, m_{\tilde{b}_{2}}^{2}, m_{\tilde{g}}^{2}\right) \\
\Delta_{b, 2}^{e l w} & =\frac{\lambda_{t}^{2}\left(\mu_{R}\right)}{(4 \pi)^{2}} A_{t} \mu I\left(m_{\tilde{t}_{1}}^{2}, m_{\tilde{t}_{2}}^{2}, \mu^{2}\right)
\end{aligned}
$$

and $\delta_{1}, \delta_{2}$ being the 2-loop corrections. The expression in Eq. 4.3 resums therefore all the contributions up to $\mathscr{O}\left(\alpha_{s}^{n+1} \mu^{n} \tan ^{n} \beta\right), \mathscr{O}\left[\alpha_{s}\left(\lambda_{t}^{2} A_{t} \tan \beta\right)^{n}\right]$ and $\mathscr{O}\left(\alpha_{s}^{n+1} A_{b}^{n}\right)$.

Similarly, the expression for the strange effective Yukawa coupling is given by

$$
\Delta_{s}=\frac{\Delta_{s, 2}\left[1+\delta_{s}\right] \tan \beta}{1+\Delta_{s, 1}\left[1+\delta_{s}\right]}
$$

where the terms of $\mathscr{O}\left[\left(\alpha_{s} \mu \tan \beta\right)^{n}\right], \mathscr{O}\left[\alpha_{s}^{n+1}(\mu \tan \beta)^{n}\right], \mathscr{O}\left[\left(\alpha_{s} A_{s}\right)^{n}\right]$ and $\mathscr{O}\left[\alpha_{s}^{n+1} A_{s}^{n}\right]$, together with all mixed terms of these orders, are resummed [3]. Notice that in this case the 1-loop EW contribution is absent $\left(\Delta_{s, 2}^{e l w}=0\right)$. The other 1-loop expressions can be obtained from Eq. 4.4 with the appropriate replacements.

\section{Application to Higgs decays into $b \bar{b}$ and $c \bar{s}$}

The partial decay width of a neutral Higgs boson $\Phi=h, H, A$ in the MSSM is given by the following expression [4]:

$$
\Gamma[\Phi \rightarrow b \bar{b}]=\frac{3 G_{F} M_{\Phi}}{4 \sqrt{2} \pi} \bar{m}_{b}^{2}\left(M_{\Phi}\right)\left[1+\delta_{\mathrm{QCD}}+\delta_{t}^{\Phi}\right] \tilde{g}_{b}^{\Phi}\left[\tilde{g}_{b}^{\Phi}+\delta_{S Q C D}^{r e m}\right]
$$

where $\bar{m}_{b}\left(M_{\Phi}\right)$ denotes the $\overline{\mathrm{MS}}$ bottom mass at the scale of the corresponding Higgs mass $M_{\Phi}$ and quark mass effects beyond $\mathscr{O}\left(m_{b}^{2}\right)$ are neglected. The corrections $\delta_{\mathrm{QCD}}+\delta_{t}$ are pure QCD corrections: the terms $\delta_{\mathrm{QCD}}$ is known up to the $\mathrm{N}^{4} \mathrm{LO}$ (leading corrections with the leading mass effects), while the top-induced $\delta_{t}$ is known up to NNLO. Compact expressions are reported in [12] and the corresponding references can be found therein. On the other hand, the factor $\tilde{g}_{b}^{\Phi}\left[\tilde{g}_{b}^{\Phi}+\delta_{S Q C D}^{r e m}\right]$ encodes the SUSY-QCD corrections: $\tilde{g}_{b}^{\Phi}$ is the resummed effective Yukawa coupling, containing the dominant part of the SUSY-QCD corrections, while $\delta_{S Q C D}^{r e m}$ is the remainder. The latter is known at NLO, because the 1-loop calculation of the Higgs decay rate to $b \bar{b}$ was calculated exactly long time ago [13], and it is known to be small in phenomenologically relevant scenarios [4].

The strange Yukawa coupling is phenomenologically relevant mainly in the charged Higgs decay into charm and strange quarks. The partial decay width is given by $[14,15]$

$$
\Gamma\left[H^{+} \rightarrow c \bar{s}\right]=\frac{3 G_{F} M_{H^{ \pm}}}{4 \sqrt{2} \pi}\left|V_{c s}\right|^{2}\left[\bar{m}_{c}^{2}\left(M_{H^{ \pm}}\right)\left(g_{c}^{A}\right)^{2}+\bar{m}_{s}^{2}\left(M_{H^{ \pm}}\right)\left(\tilde{g}_{s}^{A}\right)^{2}\right]\left(1+\delta_{\mathrm{QCD}}\right)
$$

where $\delta_{\mathrm{QCD}}$ is the same as above, $g_{s}^{A}$ is the resummed strange Yukawa coupling and the remainder is extremely small and can be safely neglected, as well as regular quark mass effects. 


\section{Conclusions}

Down-type Yukawa couplings, in particular the bottom Yukawa coupling, play an important role in MSSM scenarios characterized by large $\tan \beta$. In these Proceedings we have reviewed the calculation of the radiative corrections to the bottom and strange couplings in the MSSM and recalled how the leading parts of the SUSY-QCD corrections can be absorbed and resummed in effective Yukawa couplings.

The radiative corrections are currently known at two loops in SUSY-QCD [1-3], while the EW corrections are known at the one-loop level [4-8]. The mixed $\mathscr{O}\left(\alpha_{s} \alpha\right)$ corrections consisting in the SUSY-QCD corrections to the $\mathscr{O}(\alpha)$ one-loop contributions are in preparation and will appear soon [9].

\section{References}

[1] D. Noth and M. Spira, Phys. Rev. Lett. 101 (2008) 181801 [arXiv:0808.0087 [hep-ph]].

[2] D. Noth and M. Spira, JHEP 1106 (2011) 084 [arXiv:1001.1935 [hep-ph]].

[3] M. Ghezzi, S. Glaus, D. Müller, T. Schmidt and M. Spira, arXiv:1711.02555 [hep-ph].

[4] J. Guasch, P. Hafliger and M. Spira, Phys. Rev. D 68 (2003) 115001 [hep-ph/0305101].

[5] M. Carena, D. Garcia, U. Nierste and C. E. M. Wagner, Nucl. Phys. B 577 (2000) 88 [hep-ph/9912516].

[6] L. J. Hall, R. Rattazzi and U. Sarid, Phys. Rev. D 50 (1994) 7048 [hep-ph/9306309].

[7] R. Hempfling, Phys. Rev. D 49 (1994) 6168.

[8] M. Carena, M. Olechowski, S. Pokorski and C. E. M. Wagner, Nucl. Phys. B 426 (1994) 269 [hep-ph/9402253].

[9] M. Ghezzi, S. Glaus, D. Müller, T. Schmidt and M. Spira, arXiv:1711.02555v3, to appear.

[10] J. R. Ellis, M. K. Gaillard and D. V. Nanopoulos, Nucl. Phys. B 106 (1976) 292.

[11] M. A. Shifman, A. I. Vainshtein, M. B. Voloshin and V. I. Zakharov, Sov. J. Nucl. Phys. 30 (1979) 711 [Yad. Fiz. 30 (1979) 1368].

[12] M. Spira, Prog. Part. Nucl. Phys. 95 (2017) 98 [arXiv:1612.07651 [hep-ph]].

[13] A. Dabelstein, Nucl. Phys. B 456 (1995) 25 [hep-ph/9503443].

[14] C. S. Li and R. J. Oakes, Phys. Rev. D 43 (1991) 855.

[15] A. Mendez and A. Pomarol, Phys. Lett. B 252 (1990) 461. 